\title{
Repetições CAG: candidatos na gênese das psicoses funcionais
}

Ivanor Velloso Meira Limaำ

\section{Resumo}

O autor discorre sobre a instabilidade do DNA em regiões de repetições CAG e sua associação com doenças que afetam o SNC e apresentam o fenômeno da antecipação genética. Revisa também os achados de antecipação em famílias com transtorno bipolar e esquizofrenia, assim como as investigações com o método RED (Repeat Expansion Detection) e com o anticorpo 1C2, que apontam para uma participação desse mecanismo mutacional na determinação genética das psicoses funcionais.

\section{Descritores}

Genética das psicoses; repetições CAG; antecipação; DNA instável

\section{Abstract}

The author comments about the DNA instability associated with CNS's diseases which display genetic anticipation. He reviews the evidence of anticipation in bipolar and schizophrenia families. He also presents the RED (Repeat Expansion Detection) investigations and screenings with the antibody $1 \mathrm{C} 2$ which demonstrated the participation of this mutational mechanism on the functional psychosis genesis.

\section{Keywords}

Genetics of psychosis; CAG repeats; anticipation; unstable DNA

\section{Introdução}

A presença de um componente hereditário na etiologia das grandes psicoses funcionais - esquizofrenia e transtorno afetivo bipolar - já foi estabelecida há alguns anos através de estudos com famílias,,${ }^{1,2}$ com gêmeos, ${ }^{3,4} \mathrm{e}$ com adotados.${ }^{5,6}$ Persiste, contudo, até os dias atuais, uma indefinição sobre o modo como se dá a transmissão genética dessas enfermidades ${ }^{7,8} \mathrm{e}$, sobretudo, quais os genes envolvidos. ${ }^{9,10}$

Uma nova perspectiva de compreensão do modelo de herança dessas psicoses surgiu com a descoberta no início desta década de um novo mecanismo de mutação denominado "DNA instável".

\section{O “DNA instável” e o surgimento de doenças}

O DNA instável consiste basicamente de regiões do genoma em que um grupo de três nucleotídeos repete-se várias vezes. Em decorrência dessa disposição peculiar, nesses sítios haveria uma maior propensão à ocorrência de deslizes da enzima polimerase inserindo nucleotídeos a mais nesse segmento durante a replicação do DNA.

Essa ocorrência favoreceria a expansão do segmento de trinucleotídeos repetidos que, se localizados em genes, desencadeariam o surgimento de doenças. A expansão das repetições de trinucleotídeos foi denominada também de mutação dinâmica, pois a ocorrência da primeira mutação aumenta a chance de novos deslizes nesse sítio com mais expansões e mais deslizes ${ }^{11}$ (Figura 1).

Figura 1 - Relação direta entre o aumento das repetições trinucleotídicas e a variação da expressão fenotípica.

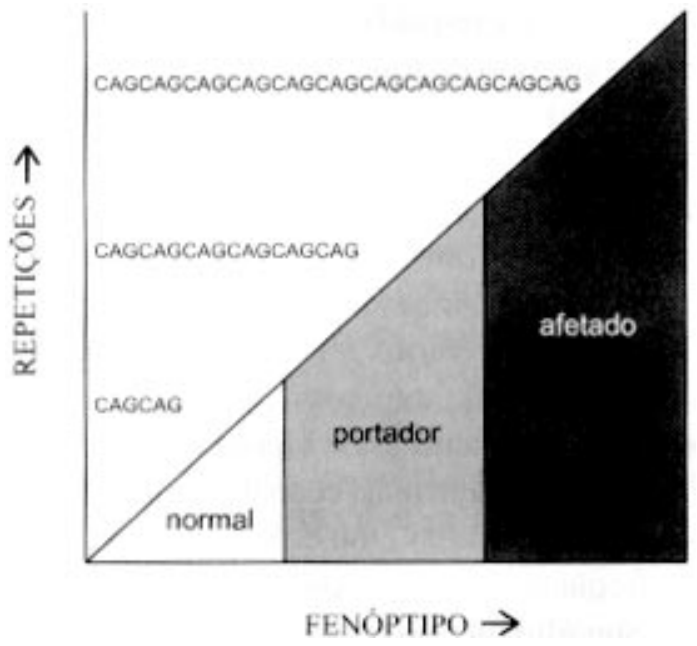

Várias doenças já foram identificadas como sendo causadas pela instabilidade do DNA em sítios com seqüências repetidas de trinucleotídeos (ver as principais na tabela 1). Nas famílias de afetados, podemos encontrar, no gene que determina a doença: indivíduos com um número de repetições similar à faixa encontrada na população geral e que, portanto, não apresen-

1. Doutorando do Departamento de Psiquiatria da Universidade Federal de São Paulo/Escola Paulista de Medicina 
Tabela 1 - Principais enfemidades determinadas por expansões de repetições trinucleotídicas.

Doenças

Síndrome do X frágil ${ }^{12}$

Doença de Kenedy (SBMA) ${ }^{13}$

Distrofia Miotônica ${ }^{14}$

Doença de Huntington ${ }^{15}$

Ataxia Espino-Cerebelar ${ }^{16}$

Doença de Machado-Joseph ${ }^{17}$

Atrofia Dentato-Rubral

e Palidolusiana ${ }^{18}$

Ataxia de Friedreich ${ }^{19}$

$\begin{array}{llrr}\text { Gene/Local } & \text { Repetição } & \text { Normal } & \text { Mutado } \\ & & & \\ \text { FMR1/Xq27.3 } & \text { CGG } & 6-54 & 230-4000 \\ \text { AR/Xq11-12 } & \text { CAG } & 11-33 & 40-66 \\ \text { MT-PK/19q13.2 } & \text { CTG } & 5-30 & 50-3000 \\ \text { IT15/4p16.3 } & \text { CAG } & 10-30 & 36-121 \\ \text { SCA1/6p22-23 } & \text { CAG } & 19-36 & 40-81 \\ \text { MJD1/14q32.1 } & \text { CAG } & 12-37 & 61-84 \\ \text { B37/12p.12 } & \text { CAG } & 7-34 & 49-75 \\ \text { X25/9q13 } & \text { GAA } & 7-22 & 200-900\end{array}$

tam a doença; indivíduos com um número discretamente aumentado dessas repetições, que seriam chamados pré-mutantes ou portadores com grande chance de terem afetados em sua prole; e, por fim, os indivíduos com um número bem maior de repetições que apresentariam o fenótipo afetado.

Um dos aspectos comuns a todas essas enfermidades é o fato de comprometerem invariavelmente o SNC e a tendência ao agravamento da doença ou ao início mais cedo dos sintomas através das sucessivas gerações, fenômeno que se denomina antecipação genética e que é hoje correlacionado ao aumento das repetições..$^{20,21}$

\section{A antecipação genética e as psicoses funcionais}

É importante ressaltar que o conceito de antecipação foi delineado ainda no começo deste século por F. W. Mott (1911) a partir da observação de 420 pares de pais e filhos afetados por distúrbios psiquiátricos nos asilos de Londres e que mostrava que o início da doença nos filhos sempre ocorria mais cedo. ${ }^{22}$

Esse achado foi posteriormente descartado pelos geneticistas como proveniente de vieses na seleção da amostra, pois não havia mecanismo biológico que respaldasse tal observação clínica. ${ }^{23} \mathrm{Com}$ a descoberta da expansão de trinucleotídeos repetidos como mecanismo biológico que explica o agravamento do quadro clínico ao longo das gerações de uma família de afetados, desenvolveu-se um renovado interesse na investigação do fenômeno nas famílias de doentes psiquiátricos com um componente genético estabelecido como esquizofrenia e transtorno bipolar.

Nos últimos seis anos, vários relatos de antecipação genéticos têm sido publicados, tanto com famílias de afetados por esquizofrenia como em famílias de bipolares, através da verificação de um início cada vez mais cedo dos sintomas nas sucessivas gerações dessas famílias e/ou do agravamento do fenótipo inferido pela freqüência de episódios, número de hospitalizações, taxas de suicídio ou utilização de uma hierarquização diagnóstica que considera quadros do espectro esquizofrênico e bipolar como formas mais leves dessas entidades (por exemplo, ciclotimia, bipolar tipo II, etc.)..$^{24-33}$

Os relatos de antecipação genética nas psicoses funcionais têm influenciado substancialmente a pesquisa molecular dessas enfermidades, e novas estratégias têm sido elaboradas na tentativa de averiguação da hipótese de que as psicoses funcionais também seriam causadas pela expansão de repetições trinucleotídicas em determinado locus gênico.

\section{Investigações moleculares do "DNA instável" nas psicoses}

Nesse caminho, investigações realizadas com o RED (Repeat Expansion Detection) - um novo método de detecção de expansões de repetições ao longo do genoma sem necessidade de conhecimento prévio de sua localização cromossômica ${ }^{34}$ - já acrescentaram novos dados a essa hipótese.

Usando o RED, vários estudos descreveram a presença de repetições CAG (citosina/adenina/guanina) de maior tamanho no DNA de portadores de esquizofrenia e de transtorno bipolar quando comparados ao conteúdo de repetições CAG no DNA dos controles saudáveis representativos da população geral ${ }^{35-41}$, embora nem todos os estudos observem a mesma associação. ${ }^{42-45}$

Esses resultados chamaram a atenção para os loci com repetições CAG - que são os trinucleotídeos mais encontrados em associação com doenças e que provavelmente estão mais representados nas regiões codificadoras dos genes - como sítios candidatos para as psicoses com determinação genética.

\section{Estudos moleculares em sítios específicos}

As investigações moleculares em loci específicos com repetições CAG ao longo do genoma não conseguiram ainda estabelecer resultados definitivos. Contudo, podemos destacar alguns achados mais recentes.

Dois estudos apontam que cerca de $90 \%$ das grandes repetições detectadas pelo RED podem ser explicadas pela expansão de repetições CAG em dois loci ${ }^{46,47}$. O primeiro denomina-se ERDA1 (Expanded Repeat Domain CAG1), localiza-se na região $17 q 21.3$ e suas seqüências flanquedoras não apresentaram homologia com nenhum gene conhecido até o momento. ${ }^{48,49} \mathrm{O}$ segundo sítio, o CTG18.1, localiza-se na região 18q21.1 e situase especificamente no terceiro íntron do gene SEF2-1b, que codifica um fator de estímulo a transcrição de genes, expresso no cérebro e em outros tecidos. ${ }^{50}$

Uma instabilidade com expansão intergeracional de repetições CAG no locus ERDA1 foi documentada numa única família com fenótipo de depressão com início na infância ${ }^{51}$. Entretanto, outros estudos nesse loci não relacionam expansões com qualquer fenótipo afetado identificável. ${ }^{49,52}$

No locus CTG18.1, uma expansão CAG muito grande ( 3.3Kb) foi detectada num único membro afetado de uma família de esquizofrênicos ${ }^{53}$ enquanto Lindblad e colaboradores (1998), investigando famílias de doentes afetivos, relataram uma associação dos alelos expandidos do locus CTG18.1 (acima de 40 repetições CAG) com a ocorrência de doença bipolar na prole. ${ }^{47}$ Outros estudos, entretanto, não conseguiram relacionar expansões nesses loci com o transtorno bipolar. ${ }^{50,52,54}$

Os achados mais significativos com relação à esquizofrenia foram encontrados após a clonagem e isolamento de um novo gene que codifica um canal de potássio regulado pelo cálcio, o hSKCa3 (human small conductance calcium-regulated potassium channel gene).$^{55}$ Os canais de potássio, por estarem envolvidos na modulação da ativação neuronal, já são candidatos funcionais aos distúrbios psiquiátricos. Além disso, esse 
gene especificamente apresenta duas seqüências de repetições CAG no primeiro exon, sendo que um deles apresenta mais variações de comprimento (polimórfica).

Chandy e colaboradores (1998) relataram um excesso dos alelos longos dessa região CAG polimórfica (> 20 repetições CAG) nos doentes esquizofrênicos. ${ }^{55}$ Outros estudos replicaram essa associação. ${ }^{56-59}$ Contudo, não foram observados os mesmos achados em algumas outras investigações com esquizofrênicos ${ }^{60-63}$ nem com portadores de transtorno bipolar. ${ }^{64,65}$

\section{Buscas por traços de poliglutaminas}

Um outro avanço nessa linha de pesquisa foi obtido com a descoberta de um anticorpo monoclonal denominado $1 \mathrm{C} 2$, que preferencialmente reage com longas cadeias de poliglutamina que seriam justamente o produto resultante da tradução das repetições CAG expandidas em determinado gene. ${ }^{66}$ Nesse caminho, dois grupos independentes relataram recentemente, através de técnica de Western Blot (método para detecção de proteínas), uma banda reativa ao $1 \mathrm{C} 2$ que corresponderia a uma cadeia de poliglutamina entre 50-55 kDA em células da linhagem linfoblástica de alguns pacientes esquizofrênicos por eles investigados ${ }^{67,68} \mathrm{e}$ ainda em outro membro das famílias de esquizofrênicos, que apresentava um quadro de transtorno afetivo. ${ }^{68}$ Uma averiguação recentemente realizada especificamente com doentes bipolares não detectou bandas imunorreativas ao anticorpo $1 \mathrm{C} 2 .{ }^{69}$

\section{Conclusão}

As evidências obtidas a partir dos achados de antecipação genética em famílias de bipolares e esquizofrênicos das investigações com o método RED e das buscas por longas cadeias de poliglutaminas com o anticorpo $1 \mathrm{C} 2$, estabelecem até o momento que as regiões de repetições trinucleotídicas CAG no genoma humano são importantes sítios candidatos a serem investigados na busca pelos genes de suscetibilidade às psicoses funcionais e já indicam fortemente que esse mecanismo de instabilidade do DNA está relacionado à vulnerabilidade genética de pelo menos um subgrupo dos doentes esquizofrênicos.

\section{Agradecimentos}

O autor é apoiado pela Fapesp, processo n 97/12454-1.

\section{Referências bibliográficas}

1. Kendler K, Gruenberg AM, Tsuang MT. Psychiatric Illness in FirstDegree Relatives of Schizophrenic and Surgical Control Patients. Arch Gen Psychiatry 1985;42:776-9.

2. Andreasen N, Rice J, Endicott J et al. Familial Rates of Affective Disorder. Arch Gen Psychiatry 1987;44:461-9.

3. Bertelsen A, Harvald B, Hauge M. A Danish Twin Study of Manic Depressive Disorders. Br J Psychiatry 1977;130:330-51.

4. McGuffin P, Farmer A, Gottesman I. Twin Concordance for Operationally Defined Schizophrenia. Arch Gen Psychiatry 1984;41:541-55.

5. Mendlewicz J, Rainer JD. Adoption Study Supporting Genetic Transmission in Manic-Depressive Illness. Nature 1977;268:327-9.

6. Wender PH, Kety SS, Rosenthal D, Schulsinger F, Ortmann J, Lunde I. Psychiatrics Disorders in the Biological and Adoptive Families of Adopte Individuals with Affective Disorders. Arch Gen Psychiatry 1986;43:923-9.

7. Rice J, Reich T, Andreasen $\mathrm{N}$ et al. The Familial Transmission of Bipolar Illness. Arch Gen Psychiatry 1987;44:441-7.

8. Pauls DL, Bailey JN, Carter AS et al. Complex Segregation Analysis of Old Order Amish Families Ascertained Through bipolar I Individuals. Am J Med Genet 1995;60(4):290-7.

9. Risch N, Botstein D. A Manic Depressive History. Nat Genet 1996;12(4):351-3.

10. Crow TJ. Current status of linkage for schizophrenia: Polygenes of vanishingly small effect or multiple false positive? Am J Med Genet 1997;74:99-103.

11. Richards RJ, Sutherland GR. Dynamic Mutations: A New Class of Mutations Causing Human Disease. Cell 1992;70:709-12.

12. Fu Y, Kuhl DPA, Pizzuti A et al. Variation of the CGG Repeat at the Fragile X Site Results in Genetic Instability: Resolution of the Sherman Paradox. Cell 1991;67:1047-58.

13. La Spada AR, Wilson EM, Lubahn DB et al. Androgen Receptor Gene Mutations in X-Linked Spinal and Bulbar Muscular Atrophy. Nature 1991;352:77-9.

14. Brook JD, Mc Currach ME, Harley HG et al. Molecular Basis of Myotonic Dystrophy: Expansion of a Trinucleotide (CTG) Repeat at the 3'End of a Transcript Encoding a Protein Kinase Family Member. Cell 1992;68:799-808.
15. The Huntington's Disease Colaborative Research Group. A Novel Gene Containing a Trinucleotide Repeat that is Expanded and Unstable on Huntington's Disease Chromosomes. Cell 1993;72:971-83.

16. OrrR HT, Chung M, Banfi S et al. Expansion of an Unstable Trinucleotide CAG repeat in Spinocerebelar Ataxia Type 1. Nat Genet 1993;4:221-6.

17. Kawaguchi Y, Yanagisawa H, Sato K et al. CAG Expansions in a Novel gene for Machado-Joseph Disease at Chromosome 14q32.1. Nat Genet 1994;8:221-8.

18. Koide R, Ikeuchi T, Onodera $\mathrm{O}$ et al. Unstable Expansion of CAG Repat in Hereditary Dentatorubral-Pallidoluysian Atrophy (DRPLA). Nat Genet 1994;6:9-13.

19. Campuzano V, Montermini L, Molto MD et al. Friedreich's Ataxia: Autosomal Recessive Disease Caused by an Intronic GAA Triplet Repeat Expansion. Science 1996;271:1423-7.

20. Sutherland GR, Richards RJ. Anticipation Legitimized: Unstable DNA to the Rescue. Am J Hum Genet 1992;51:7-9.

21. Lindblad K, Schalling M. Clinical implications of unstable DNA repeat sequences. Acta Paediatr 1996;85:256-71.

22. Mott FW. A Lecture on Heredity and Insanity. Lancet 1911;1:1251-9.

23. Penrose L. The Problem of Anticipation in Pedigrees of Myotonic Dystrophy. Ann Eugenics 1948;14:125-32.

24. McInnis MG, McMahon FJ, Chase GA et al. Anticipation in Bipolar Affective Disorder. Am J Hum Genet 1993;53:385-90.

25. Basset AS, Honer WG. Evidence for Anticipation in Schizophrenia. Am J Hum Genet 1994;54:864-70.

26. Nylander PO, Engströn $C$, Chotai $J$ et al. Anticipation in Swedish Families with Bipolar Affective Disorder. J Med Genet 1994;31:686-9.

27. Thibaut F, Martinez M, Petit M et al. Futher Evidence for Anticipation in Schizophrenia. Psychiatry Res 1995;59:25-33.

28. Gorwood P, Leboyer M, Falissard B et al. Anticipation in Schizophrenia: New ligth on a Controversial Problem. Am J Psychiatry 1996;153:1173-7.

29. Johnson JE, Cleary J, Ahsan H et al. Anticipation and Schizophrenia: Biology or Bias? Am J Med Genet 1997;74:275-80.

30. Serbanescu-Grigoroiu M, Wickramatne PJ, Hodge S et al. Genetic Anticipation and Imprinting in Bipolar I Illness. Br J Psychiatry 1997;170:162-6 
31. Ohara K, Suzuki Y,Yoshida K et al. Anticipation and Imprinting in Japanese Familial Mood Disorders. Psychiatry Res 1998;79(3):1918.

32. Valero J, Martorell L, Marine $\mathrm{J}$ et al. Anticipation and imprinting in Spanish Families with Schizophrenia. Acta Psychiatr Scand 1998;97(5):343-50.

33. Heiden A, Willinger U, Scharfetter $\mathbf{J}$ et al. Anticipation in Schizophrenia. Schizophr Res 1999;35(1):25-32.

34. Schalling M, Hudson TJ, Bustow KH et al. Direct Detection of Novel Expanded Trinucleotide Repeat in the Human Genome. Nat Genet 1993;4:135-9.

35. O’Donovan MC, Guy C, Craddock N et al. Expanded CAG Repeats in Schizophrenia and Bipolar Disorder. Nat Genet 1995;10:380-1.

36. Lindblad K, Nylander PO, De Bruyn A et al. Detection of Expanded CAG Repeats in Bipolar Affective Disorder Using the Repeat Expansion Detection (RED) Method. Neurobiol Dis 1995;2:5562

37. Morris AG, Gaitonde E, McKenna PJ et al. CAG Repeat Expansions and Schizophrenia - Association with Disease in Females and Early Age-at-onset. Hum Mol Genet 1995;4:1957-61.

38. O'Donovan MC, Guy C, Craddock N et al. Confirmation of Association Between Expanded CAG/CTG Repeats and Both Schizophrenia and Bipolar Disorder. Psychol Med 1996;26:114553.

39. Oruc L, Lindblad K, Verheyen GR et al. CAG Repeat Expansions in Bipolar and Unipolar Disorder. Am J Hum Genet 1997;60:730-

40. Mendlewicz J, Lindblad K, Souery D et al. Expanded trinucleotide CAG repeats in families with bipolar affective disorder. Biol Psychiatry 1997;42(12):1115-22.

41. Burgess CE, Lindblad K, Sidransky E et al. Large CAG/CTG repeats are associated with childhood-onset schizophrenia. Mol Psychiatry 1998;3(4):321-7.

42. Vincent JB, Klempan T, Parikh SS et al. Frequency Analysis of Large CAG/CTG Trinucleotide Repeats in Schizophrenia and Bipolar Affective Disorder. Mol Psychiatry 1996;1(2):141-8.

43. Li, T, Vallada HP, Liu X et al. Analysis of CAG/CTG repeat size in Chinese subjects with schizophrenia and bipolar affective disorder using the repeat expansion detection method. Biol Psychiatry 1998;44(11):1160-5.

44. Zander C, Schurhoff F, Laurent $\mathrm{C}$ et al. CAG repeat sequences in bipolar affective disorder: no evidence for association in a French population. Am J Med Genet 1998;81(4):338-41.

45. Martorell L, Pujana MA, Valero J et al. Anticipation is not associated with CAG repeat expansion in parent-offspring pairs of patients affected with schizophrenia. Am J Med Genet 1999;88(1):50-6.

46. Sidransky E, Burgess C, Ikeuchi T et al. A Triplet Repeat on $17 \mathrm{q}$ Accounts for Most Expansions Detected by the Repeat-Expansion Detection Technique. Am J Hum Genet 1998;62:1148-51.

47. Lindblad K, Nylander P-O, Zander C et al. Two commonly expanded CAG/CTG repeat loci: involvement in affective disorders? Mol Psychiatry 1998;3(5):405-10.

48. Nakamoto M, Takebayashi H, Kawaguchi $\mathrm{Y}$ et al. A CAG/CTG Expansion in the Normal Population. Nat Genet 1997;17:385-6.

49. Ikeuchi T, Sanpei K, Takano H et al. A Novel Long and Unstable CAG/CTG Trinucleotide Repeat on Chromosome 17q. Genomics 1998;49:321-6.

50. Breschel TS, McInnis M, Margolis R et al. A novel, heritable, expanding CTG repeat in an intron of the SEF2-1 gene on chromosome 18q21.1. Hum Mol Genet 1997;6(11):1855-63.

51. Vincent JB, Kovacs M, Krol R et al. Intergenerational CAG repeat expansion at ERDA1 in a family with childhood-onset depression, schizoaffective disorder, and recurrent major depression. Am J Med Genet 1999a;88(1):79-82.

52. Guy CA, Bowen T, Jones I et al. CTG18.1 and ERDA-1 CAG/ CTG Repeat Size in Bipolar Disorder. Neurobiol Dis 1999;6(4):302-7.

53. Sirugo G, Pakstis AJ, Kidd K et al. Detection of a large CTG/CAG trinucleotide repeat expansion in a Danish schizophrenia kindred. Am J Med Genet 1997;74(5):546-8.

54. Vincent JB, Petronis A, Strong E et al. Analysis of genome-wide CAG/CTG repeats, and at SEF2-1B and ERDA1 in schizophrenia and bipolar affective disorder. Mol Psychiatry 1999b;4(3):22934.

55. Chandy KG, Fantino E, Wittekindt $\mathrm{O}$ et al. Isolation of a novel potassium channel gene hSKCa3 containing a polymorphic CAG repeat: a candidate for schizophrenia and bipolar disorder? Mol Psychiatry 1998;3(1):32-7.

56. Bowen T, Guy C, Craddock et al. Further support for an association between a polymorphic CAG repeat in the hKCa3 gene and schizophrenia. Mol Psychiatry 1998;3(3):266-9.

57. Wittekindt O, Jauch A, Burgert E et al. The Human Small Conductance Calcium-Regulated Potassium Channel Gene (hSKCa3) Contains Two CAG Repeats in Exon 1, is on Chromosome 1q21.3, and Shows a Possible Association with Schizophrenia. Neurogenetics 1998;1:259-65.

58. Cardno AG, Bowen T, Guy CA et al. CAG repeat length in the hKCa3 gene and symptom dimensions in schizophrenia. Biol Psychiatry 1999;45(12):1592-6.

59. Dror V, Shamir E, Ghanshani S et al. hKCa3/KCNN3 potassium channel gene: association of longer CAG repeats with schizophrenia in Israeli Ashkenazi Jews, expression in human tissues and localization to chromosome 1q21. Mol Psychiatry 1999;4(3):254-60.

60. Li T, Hu X, Chandy KG et al. Transmission disequilibrium analysis of a triplet repeat within the $\mathrm{hKCa} 3$ gene using family trios with schizophrenia. Biochem Biophys Res Commun 1998;251(2):662-5.

61. Stober G, Jatzke S, Meyer J et al. Short CAG repeats within the hSKCa3 gene associated with schizophrenia: results of a familybased study. Neuroreport 1998;9(16):3595-9.

62. Antonarakis SE, Blouin JL, Lasseter VK et al. Lack of linkage or association between schizophrenia and the polymorphic trinucleotide repeat within the KCNN3 gene on chromosome $1 \mathrm{q} 21$. Am J Med Genet 1999;88(4):348-51.

63. Joober R, Benkelfat $\mathrm{C}$, Brusebois $\mathrm{K}$ et al. Lack of association between the hSKCa3 channel gene CAG polymorphism and schizophrenia. Am J Med Genet 1999;88(2):154-7.

64. Guy CA, Bowen T, Williams $\mathrm{N}$ et al. No association between a polymorphic CAG repeat in the human potassium channel gene hKCa3 and bipolar disorder. Am J Med Genet 1999;88(1):57-60.

65. McInnis MG, Breschel T, Margolis R, et al. Family-Based Association Analysis of the hSKCa3 Potassium Channel Gene in Bipolar Disorder. Mol Psychiatry 1999;4(3):217-9.

66. Ross CA. Schizophrenia genetics: expansion of knowledge? Mol Psychiatry 1999;4(1):4-5.

67. Moriniere S, Saada C, Holbrt S et al. Detection of polyglutamine expansion in a new acidic protein: a candidate for childhood onset schizophrenia? Mol Psychiatry 1999;4(1):58-63.

68. Joober R, Benkelfat C, Jannatipour $M$ et al. Polyglutaminecontaining proteins in schizophrenia. Mol Psychiatry 1999;4(1):537.

69. Turecki G, Alda M, Grof P et al. Polyglutamine Tracts: No Evidence of a Major Role in Bipolar Disorder. Mol Psychiatry 1999;4(3):220-1.

Correspondência: Ivanor Velloso Meira Lima

Departamento de Psiquiatria - Escola Paulista de Medicina Rua Botucatu, 740 - $3^{\circ}$ andar

Vila Clementino São Paulo - SP 04023-900 Email: Ivanorlima@psiquiatria.epm.br 